\title{
Solving a Dynamic Real-Life Vehicle Routing Problem
}

\author{
Asvin Goel and Volker Gruhn \\ Chair of Applied Telematics and e-Business, Computer Science Faculty, University of \\ Leipzig, Klostergasse 3, 04109 Leipzig, Germany \\ \{goel, gruhn\} @ebus.informatik.uni-leipzig.de
}

\begin{abstract}
Summary. Real-life vehicle routing problems encounter a number of complexities that are not considered by the classical models found in the vehicle routing literature. In this paper we consider a dynamic real-life vehicle routing problem which is a combined load acceptance and generalised vehicle routing problem incorporating a diversity of practical complexities. Among those are time window restrictions, a heterogeneous vehicle fleet with different travel times, travel costs and capacity, multi-dimensional capacity constraints, order/vehicle compatibility constraints, orders with multiple pickup, delivery and service locations, different start and end locations for vehicles, route restrictions associated to orders and vehicles, and drivers' working hours. We propose iterative improvement approaches based on Large Neighborhood Search. Our algorithms are characterised by very fast response times and thus, can be used within dynamic routing systems where input data can change at any time.
\end{abstract}

\section{Introduction}

In this paper we present algorithms for solving a dynamic real-life problem. The problem incorporates various practical complexities among which some have received only little attention in the vehicle routing literature. The problem is dynamic and information can change during the transportation process. We propose iterative improvement approaches based on Large Neighborhood Search. The algorithms we present are characterised by two features: they are capable of handling the practical complexities and they have very fast response times and thus, are suitable for dynamic optimisation.

\section{Problem Formulation}

This work is motivated by a practical problem arising in air-cargo transport. Most of the air-cargo within Europe is transported by so-called road feeder services (RFS), that is the transport is done on roads, see [8]. In the problem considered not all transportation requests are known before load acceptance and planning starts. Instead, transportation requests may become known at any time. In contrast to many 
other commonly known routing problems not all transportation requests have to be assigned to a vehicle, instead a so-called make-or-buy decision is necessary to determine whether a transportation request should be assigned to some vehicle (make) or not (buy).

A transportation request is specified by a nonempty set of locations which have to be visited in a particular sequence by the same vehicle, the time windows in which these locations have to be visited, and the revenue gained when the transportation request is served. Furthermore, some characteristics can be specified which constrain the possibility of assigning the transportation requests to certain vehicles due to compatibility constraints and capacity constraints. At each of the locations some shipment(s) with several describing attributes can be loaded or unloaded.

A fleet of heterogeneous vehicles is available to serve the transportation requests. The vehicles can have different capacities, as well as different travel times and travel costs between locations. The vehicles can transport shipments which require some of the capacity the vehicle supplies. Instead of assuming that each vehicle becomes available at a central depot, each vehicle is given a start location where it becomes available at a specific time and with a specific load. Furthermore, the vehicles do not have to return to a central depot and for each vehicle a final location is specified, which has to be reached within a specific time and with a specific load. Each vehicle may have to visit some locations in a particular sequence between leaving its start and reaching its final location. All locations have to be visited within a specific time window. If the vehicle reaches one of these locations before the begin of the time window, it has to wait. At each of these locations some shipment(s) may have to be loaded or unloaded. Drivers' working hours are regulated by EU Council Regulation No 3820/85. After a certain amount of driving an obligatory daily rest period is necessary before the driver(s) may continue driving. The maximal time allowed driving between two consecutive daily rest periods depends on whether a vehicle is manned by one or two drivers.

A tour of a vehicle is a journey in accordance with EU social legislation starting at the vehicles start location and ending at its final location, passing all other locations the vehicle has to visit in the correct sequence, and passing all locations belonging to each transportation request assigned to the vehicle in the correct respective sequence. A tour is feasible if and only if for all orders assigned to the tour compatibility constraints hold and at each point in the tour time window and capacity restrictions hold. The objective is to find distinct feasible tours maximising the profit, which is determined by the accumulated revenue of all served transportation requests, reduced by the accumulated costs for operating these tours.

\section{Related work}

The dynamic real-life problem discussed in this paper is a generalisation of the vehicle routing problem (VRP) and the pickup and delivery problem (PDP), see [3], and [12] and secondary literature given there. Some of the generalisations have been discussed by [7], however, no model in literature considers all aspects of the RFS 
problem. Several extensions of the VRP have been widely studied in previous works, as the VRP with time windows, see [2], and the capacitated VRP, see [10]. In many cases it is assumed that load is accepted before planning begins and tours are generated assuming that all accepted transportation requests must be served. Work regarding load acceptance issues for the travelling salesman problem (TSP) has been surveyed by [5], but only few attempts have been made to tackle extensions of this problem. Although some work addresses certain aspects of the complexities resulting from restrictions to drivers' working hours, see [15], the only work known to the authors explicitly regarding drivers' working hours is given by [17]. A comprehensive discussion of dynamic vehicle routing can be found in [13] and [14]. Dynamic real-life problems often require rich models, in most of the literature on dynamic routing problems however, some simplifying assumptions are made. The dynamic full-truckload pickup and delivery problem for example has been studied by [6] and [18]. The only work known to the authors regarding rich VRP with multiple pickup and delivery locations in a dynamic context is presented by [15].

\section{Large Neighborhood Search}

Large Neighborhood Search (LNS) has been introduced for the VRP with time windows by [16] and can be interpreted as a special case of Iterated Local Search, described in [11]. The LNS method starts with an initial solution $s$. In each iteration $k$ transportation requests are removed from their tours in the current solution $s$. A new solution $s^{*}$ is then generated by inserting unscheduled transportation requests. The new solution is accepted as the next current solution if the objective value is improved. The number of removals can be adjusted before the next iteration. If no termination criterion is fulfilled, the algorithm continues with the next iteration.

\section{LNS algorithm}

0. $s:=$ initialsolution()

1. $s^{\prime}:=$ removeorders $(s, k)$

2. $s^{*}:=\operatorname{insertorders}\left(s^{\prime}\right)$

3. if $s^{*}$ is better than $s$ set $s:=s^{*}$

4. adjust parameters

5. goto 1 or stop

[9] have shown that LNS is well suited for the VRP with several additional constraints. We will show that LNS can also be used to solve the dynamic real-life problem considered in this paper. To ensure very fast response times we propose fast insertion methods.

The first method is a sequential insertion method. In the sequential insertion method unscheduled transportation requests are randomly chosen and all feasible and efficient insertion possibilities are determined. We assume an infinite incremental cost if no feasible insertion is possible and say that an insertion possibility is efficient if the incremental cost is smaller than the revenue of the order. If an effi- 
cient insertion possibility is found the transportation request is inserted to a tour with high efficiency.

The second insertion method is based on the auction method for the vehicle routing problem with time windows by [1]. This method is illustrated in figure 1. In the first phase all unscheduled orders request and receive from each vehicle an insertion possibility and the efficiency of insertion. In the second phase each unscheduled order, which did receive an efficient insertion possibility, chooses a vehicle with low incremental costs and sends a proposal for insertion to this vehicle. In phase three each vehicle which received a proposal chooses an order for insertion to the tour. The method stops if no order can be efficiently inserted and continues otherwise.

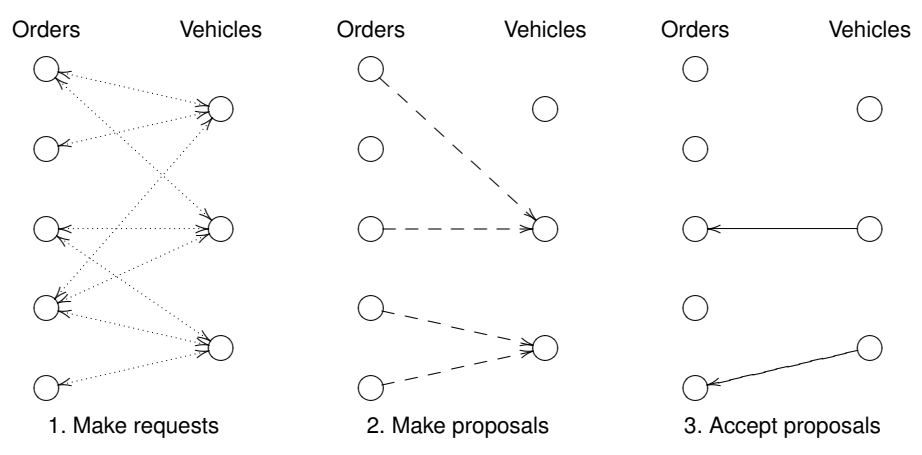

Fig. 1. Illustration of the auction method

For step 1 of the LNS algorithm [16] propose to only unschedule transportation requests which are related to each other. For the VRP a relatedness measure based on geographical closeness of customer locations has been applied to increase the opportunity for the reinsertion to achieve some improvement in the schedule. A concept similar to geographical closeness in the VRP however, does not exist for vehicle routing problems with pickups and deliveries. Thus, geographical closeness cannot be used for the problem considered in this work and transportation requests are unscheduled randomly in step 1 of the LNS algorithm.

\section{Computational experiments}

Computational experiments were performed on test cases derived from the real-life problem. We generated test problem with $|\mathcal{V}|$ vehicles and $\left|\mathcal{O}_{0}\right|$ orders which are known at the beginning of the planning horizon. In every hour of the planning horizon of one week $\left|\mathcal{O}_{t}\right|$ new orders become known. We generated a heterogeneous vehicle fleet and transportation requests with different requirements to the vehicles and pickup and delivery locations distributed as indicated in figure 2 . The length of all time windows at the locations is denoted by $\tau$. 


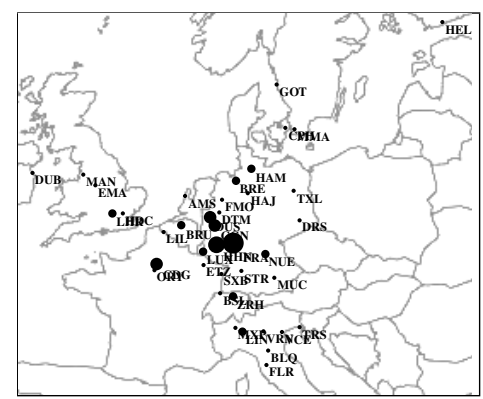

\begin{tabular}{|rrrr|r|r|r|}
\hline \multicolumn{3}{|c|}{ Problem } & \multicolumn{3}{|c|}{ Solver } \\
$|\mathcal{V}|$ & $\left|\mathcal{O}_{0}\right|$ & $\left|\mathcal{O}_{t}\right|$ & $\tau$ & LNS-A & LNS-AS & LNS-S \\
\hline 50 & 150 & 5 & $2 \mathrm{~h}$ & 53.54 & 57.93 & 54.67 \\
100 & 300 & 10 & $2 \mathrm{~h}$ & 41.42 & 39.52 & 34.03 \\
250 & 750 & 25 & $2 \mathrm{~h}$ & 24.30 & 21.42 & 17.19 \\
500 & 1500 & 50 & $2 \mathrm{~h}$ & 13.19 & 9.49 & 8.77 \\
50 & 150 & 5 & $12 \mathrm{~h}$ & 58.57 & 49.92 & 54.49 \\
100 & 300 & 10 & $12 \mathrm{~h}$ & 39.00 & 35.53 & 32.37 \\
250 & 750 & 25 & $12 \mathrm{~h}$ & 29.50 & 24.73 & 25.38 \\
500 & 1500 & 50 & $12 \mathrm{~h}$ & 13.75 & 13.06 & 9.38 \\
\hline
\end{tabular}

Fig. 2. Problem characteristics and results

At each timestep all transportation requests which were unscheduled were permanently rejected before new transportation requests were added to the problem. New transportation requests were inserted to the tours by the auction method afterwards. The solution obtained hereby was used as a reference solution. To test our algorithms we only allowed 30 seconds of computation time per timestep on a personal computer with Intel Pentium 4 processor with $3.00 \mathrm{GHz}$ and linux operating system. The average time per iteration of our LNS algorithms was below one second for all test problems except for those with 500 vehicles where the average time per iteration was below 1.75 seconds. In figure 2 we show the percentage of improvement over the reference solutions. The LNS method using the auction method for reinsertion is denoted by LNS-A, the LNS method using the sequential method for reinsertion is denoted by LNS-S. The LNS method denoted by LNS-AS randomly switches between the sequential and the auction method. We can see that in most cases LNS-A outperforms the other algorithms. As diversification is high, the sequential insertion methods LNS-S and LNS-AS can also in certain cases produce very good results. No significant changes in the performance of our algorithms can be identified between the test cases with very short time windows and longer time windows.

\section{Conclusions}

In this paper we considered a dynamic real-life problem which is a combined load acceptance and vehicle routing problem. The problem incorporates some practical complexities which received only little attention in the vehicle routing literature. We presented algorithms based on Large Neighborhood Search which are capable of handling these complexities. Our computational experiments have shown that the algorithms perform well for problems with hundreds of vehicles and several hundreds of transportation requests and response times were often less than a second. The combination of fast response times and the capability of handling the practical complexities allows the use of our algorithms in dynamic routing systems. 


\section{References}

1. Antes J and Derigs U (1995). A new parallel tour construction algorithm for the vehicle routing problem with time windows. Department of Information Systems and Operations Research, University of Cologne, Cologne, Germany.

2. Cordeau J-F, Desaulniers G, Desrosiers J, Solomon MM, and Soumis F (2002). VRP with time windows. In P. Toth and D. Vigo, editors, The Vehicle Routing Problem, pages 157-193. SIAM Monographs on Discrete Mathematics and Applications, Philadelphia.

3. Cordeau J-F, Gendreau M, Hertz A, Laporte G, and Sormany J-S (2004). New heuristics for the vehicle routing problem. Les cahiers du GERAD G-2004-33, Université de Montreal HEC, Montréal, Canada.

4. Council of the European Communities (1985). Council Regulation (EEC) No 3820/85 of 20 December 1985 on the harmonization of certain social legislation relating to road transport.

5. Feillet D, Dejax P, and Gendreau M (2005). Traveling Salesman Problems with Profits. Transportation Science, 39(2):188-205.

6. Fleischmann B, Gnutzmann S, and Sandvoß E (2004). Dynamic vehicle routing based on on-line traffic information. Transportation Science, 38(4):420-433.

7. Hasle G (2003). Heuristics for rich VRP models. Presented at the Seminar at GERAD, 30.10.2003, Montréal, Canada.

8. Heckmann M (2002). DV-gestütztes Geschäftsprozeßmanagement in der Luftfrachtlogistik. Dissertation, Shaker Verlag Aachen.

9. Kilby P, Prosser P, and Shaw P (2000). A comparison of traditional and constraint-based heuristic methods on vehicle routing problems with side constraints. Constraints, 5:389414.

10. Laporte G and Semet F (2002). G. Classical heuristics for the capacitated VRP. In Toth P and Vigo D, editors, The Vehicle Routing Problem, pages 109-128. SIAM Monographs on Discrete Mathematics and Applications, Philadelphia.

11. Lourenço H, Martin O, and Stützle T (2002). Iterated Local Search. In Glover F and Kochenberger G, (eds), Handbook of Metaheuristics. Kluwer, pp 321-353.

12. Mitrović-Minić S (1998). Pickup and delivery problem with time windows: A survey. Technical report TR 1998-12, School of Computing Science, Simon Fraser University, Burnaby, BC, Canada.

13. Psaraftis HN (1988). Dynamic vehicle routing problems. In B.L. Golden and A.A. Assad, editors, Vehicle routing: Methods and studies, pages 233-248. North-Holland Amsterdam.

14. Psaraftis HN (1995). Dynamic vehicle routing: Status and prospects. Annals of Operations Research, 61:143-164.

15. Savelsbergh MWP and Sol M (1998). DRIVE: dynamic routing of independent vehicles. Operations Research, 46:474-490.

16. Shaw $P$ (1997). A new local search algorithm providing high quality solutions to vehicle routing problems. Technical report, APES group, Department of Computer Sciences, University of Strathclyde, Glasgow, Scottland.

17. Xu H, Chen Z-L, Rajagopal S, and Arunapuram S (2003). Solving a practical pickup and delivery problem. Transportation Science, 37(3):347-364.

18. Yang J, Jaillet P, and Mahmassani H (2004). Real-time multi-vehicle truckload pickupand-delivery problems. Transportation Science, 38(2):135-148. 